\title{
CFTR contributes to the development of hypertension by affecting MLC phosphorylation via $\mathrm{Ca}^{2+}$-dependent and $\mathrm{Ca}^{2+}$-independent pathways
}

\section{Liyan Zhao ${ }^{1,2}$, Xiao Chen ${ }^{1}$, Yongyuan Guan², Guanlei Wang ${ }^{2}$}

${ }^{I}$ The First Affiliated Hospital of Sun Yat-sen University, China, ${ }^{2}$ Zhongshan School of Medicine, Sun Yat-sen University, China

Background: The arterial vasoconstriction is a determinant of blood pressure (BP) control and is dependent on the phosphorylation of myosin light chain (MLC). Cystic fibrosis transmembrane conductance regulator (CFTR) functions as a cAMP-dependent chloride channel and has been linked to the regulation of vascular tone and clinical hypotension. In the presented study we investigated whether CFTR was involved in the development of hypertension, and explored the underlying mechanisms.

Method: Wild type (WT), CFTR knockout mice and WT rats were implanted with mini-osmotic pumps loaded with Ang II to establish the Ang-II induced hypertension model. BP was measured every 3 days using tail-cuff blood pressure system. The basilar artery or aorta rings were examined by the myography. The level of CFTR mRNA was examined by qPCR and the protein expression of phosphorylated MLC was detected using western blotting and IFC. The intracellular calcium concentration $\left(\left[\mathrm{Ca}^{2+}\right]_{\mathrm{i}}\right)$ in cultured basilar arterial smooth muscle cells (BASMCs) was analyzed using Fura-2 AM. Results: Compared with WT mice infused with Ang II, both SBP and DBP of CFTR knockout mice were significantly increased during the treatment. CFTR mRNA in basilar artery of mice was negatively related with blood pressure. Next, the selective CFTR inhibitor Cir-172 inhibition or genetic loss of CFTR enhanced the arterial tension induced by $\mathrm{KCl}$ $(100 \mathrm{mM})$ or AngII (100 uM), but not by 5-HT, U46619. In addition, the level of phosphorylated MLC was increased by the CFTR inhibitor or gene knockdown as well, suggesting that CFTR contributed to the regulation of vascular tone and the BP control during hypertension. The CFTR inhibitor or gene knockdown increased $\mathrm{Ca}^{2+}$ influx in response to $\mathrm{KCl}$ or Ang II without influencing basal $\left[\mathrm{Ca}^{2+}\right]_{\mathrm{i}}$. On the other hand, CFTR knockdown enhanced the $\mathrm{Ca}^{2+}$ non-dependent RhoA activity, and in turn the phosphorylation of MYPT-1 at the site of Thr850.

Conclusion: These data demonstrate that CFTR is critical for the development of Ang II-induced hypertension. CFTR regulates basilar arterial vasoconstriction and blood pressure via affecting MLC phosphorylation, which is regulated by $\mathrm{Ca}^{2}$ ${ }^{+}$-dependent and $\mathrm{Ca}^{2+}$-independent RhoA pathways. This work has revealed CFTR as a new potential therapeutic target in hypertension. 